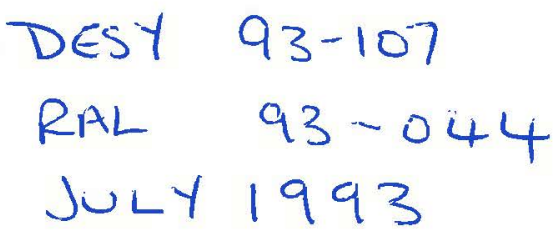

\title{
Photoproduction of multi-jet events at HERA: a Monte Carlo simulation
}

\author{
J M Butterworth $\dagger$ and I R Forshaw $\ddagger$ \\ † Laboratory for Elementary Particle Science, Pennsylvania State University, University Park, \\ PA 16802, USA \\ $\ddagger$ Theoretical Particle Physics Group, Rutherford Appleton Laboratory, Chilton, Didcot, Oxon, \\ OX11 0QX, UK
}

\begin{abstract}
We study the regime of high-energy photoproduction, currently under exploration at the DESY ep collider, HERA. In particular we discuss the possible production of more than one pair of 'back-to-back' jets which may occur at reasonably high $p_{T}$ as a consequence of the high-parton-density regime opened up at HERA centre-of-mass energies. We describe the construction of a multi-jet event generator based upon leading-order @ an eikonal formaltsm, and show that the effect of multiple parton interactions on event shapes at HERA could indeed be significant.
\end{abstract}

\section{Introduction}

The high centre-of-mass (CM) energies of the HERA and Tevatron colliders allows a study of the high-parton-density regime of QCD: a regime of great theoretical interest [1]. In this note, we explore the possibility that HERA might produce events containing more than one pair of back-io-back high- $p_{\mathrm{T}}$ jets, which could arise as a direct consequence of the multiple hard parton-parton scalters which can occur in high-parton-density collisions.

We use a simple eikonal formalism, which is described in section 2, to model the multijet production. In section 3 , we discuss the event generation prescription in some detail and in section 4 our first results are presented. Section 5 is reserved for our comments and conclusions.

\section{The eikonal formalism}

For a general review of the eikonal formalism in $\gamma p$ reactions, we refer to [2-4]. Here we briefly review the pertinent features. 

When the $\gamma p \mathrm{CM}$ energy is large enough, i.e. $s_{\gamma p} \gg p_{T}^{2}\left(p_{T}\right.$ is the jet transverse momentum), the perturbative jet cross section can be large (since the minimum parton momentum fraction probed is $\sim p_{T}^{2} / s$ and the parton distribution functions rise rapidly with decreasing momentum fraction, $x$ ). The large number of small- $x$ partons which are able to undergo hard scattering means that the probability of there being more than one scatter per $\gamma p$ interaction becomes significant. The eikonal formalism is used to quantify this physics and regularize the rise in the total $\gamma p$ cross section with $s_{\gamma p \text {. The formalism }}$ also has implications for event topologies, which we examine here. A different multiple scattering formalism is available in the PYTHIA generator, and its effect on event shapes in proton-antiproton collisions was investigated in [5].

We will need to work in impact parameter space, and we define a function $A(b)$ to determine the area overlap of partons from the colliding particles. For example, $A(b)$ is largest for 'head-on' collisions (i.e. $b=0$ ) and falls away rapidly as $b$ rises. For $A(b)$, we take a convolution of the (Fourier-transformed) form factors of the colliding particles and assume the photon to be described by vector meson dominance (VMD), i.e. its distribution of electric charge is that of a typical vector meson. In the region of small $x$ this is expected to be a reasonable assumption.

It is assumed that successive hard scatters are produced incoherently and consequently that we can use Poisson statistics to model the interaction. Defining an event as a single hard scatter (i.e. producing two jets), we use the relation

$$
\text { number of trials } \times \text { event probability per trial }=\sigma^{Y P}\left(z s, p_{\mathrm{T} 1}^{2}, p_{\mathrm{T} 2}^{2}\right) A(b) / P_{\mathrm{VMD}}
$$

as our basic building block. The cross section $\sigma^{\gamma p}\left(z s, p_{T 1}^{2}, p_{T 2}^{2}\right)$ is that for jet production in the range $p_{\mathrm{Tl}} \leqslant p_{\mathrm{T}} \leqslant p_{\mathrm{T} 2}$, and is given by

$$
\sigma^{\gamma P}\left(z s, p_{T 1}^{2}, p_{T 2}^{2}\right)=\int_{p_{T 1}^{2}}^{p_{T 2}^{2}} d p_{T}^{2} \frac{\mathrm{d} \sigma^{Y P}\left(p_{T}^{2}, z s\right)}{\mathrm{d} p_{T}^{2}}
$$

where

$$
\frac{\mathrm{d} \sigma^{\gamma \mathrm{P}}\left(p_{T}^{2}, z s\right)}{\mathrm{d} p_{T}^{2}}=\int_{4 p_{T}^{2} /(z s)}^{1} \mathrm{~d} x_{\mathrm{p}} \int_{4 p_{T}^{2} /\left(2 x_{\mathrm{p}} s\right)}^{1} \mathrm{~d} x_{y} \frac{\mathrm{d} \sigma^{\gamma \mathrm{P}}\left(x_{\mathrm{p}}, x_{y}, p_{T}^{2}\right)}{\mathrm{d} x_{\mathrm{p}} \mathrm{d} x_{y} \mathrm{~d} p_{T}^{2}}
$$

The longitudinal momentum fraction of the incoming electron carried by the photon is $z$, i.e. $s_{\gamma \mathrm{p}}=z s$ where $s$ is the ep CM energy. The factor $P_{\mathrm{VMD}}$ is the probability that the photon interacts as a vector meson, and is expected to be $\sim 1 / 300$. The extraction of this factor is necessary since we will only be considering multiple scattering once the photon can be regarded as a non-perturbative bound state. Poisson statistics then dictates that the probability of having a hard scatter in the range $p_{T 1} \leqslant p_{T} \leqslant p_{T 2}$ is

$$
=1-\exp \left(-\frac{A(b)}{P_{\mathrm{VMD}}} \sigma^{Y P}\left(z s, p_{\mathrm{T} 1}^{2}, p_{\mathrm{T} 2}^{2}\right)\right) .
$$

We can now build up an event of $n$ hard scatters. The probability for such an event to occur is equal to the probability of having a single hard scatter somewhere in the available phase space and no hard scatter with larger $p_{T}$, multiplied by the probability of having the next hard scatter with $p_{T}$ less than the first and no scatter with $p_{T}$ between it and that of the first scatter, and so on. Mathematically, the fully differential cross section for 
producing a $2 n$-jet event with the $i$ th jet originating from a parton-pair produced backto-back with transverse momentum $p \gamma_{i}$ and associated initiating partons of longitudinal momentum fractions $x_{\mathrm{pi}}$ and $x_{y i}$ is given by

$$
\begin{aligned}
& \frac{\mathrm{d} \sigma^{\mathrm{eP}}\left(\left\{x_{\mathrm{p} i}\right\},\left\{x_{y i}\right\},\left\{p_{\mathrm{T} i}^{2}\right\}, z, b, s\right)}{\mathrm{d} z \mathrm{~d}^{2} b \prod_{i=1}^{n} \mathrm{~d} x_{\mathrm{p} i} \mathrm{~d} x_{\gamma i} \mathrm{~d} p_{T i}^{2}}=P_{\mathrm{VMD}} f_{\gamma / \mathrm{e}}\left(z, E^{2}\right) \\
& \quad \times \prod_{i=1}^{n}\left[\frac{A(b)}{P_{\mathrm{VMD}}} \frac{\mathrm{d} \sigma^{\gamma \mathrm{P}}\left(x_{\mathrm{p} i}, x_{y i}, p_{T i}^{2}\right)}{\mathrm{d} x_{\mathrm{p} i} \mathrm{~d} x_{\gamma i} \mathrm{~d} p_{\mathrm{T} i}^{2}} \exp \left(-\frac{A(b)}{P_{\mathrm{VMD}}} \int_{p_{T}^{2}}^{p_{T(i-1)}^{2}} \mathrm{~d} p_{\mathrm{T}}^{2} \frac{\mathrm{d} \sigma^{\gamma \mathrm{P}}\left(p_{T}^{2}, z s\right)}{\mathrm{d} p_{\mathrm{T}}^{2}}\right)\right] .
\end{aligned}
$$

The splitting function, $f_{Y / e}\left(z, E^{2}\right)$, gives the photon momentum distribution. For the first hard scatter, $p_{\mathrm{T} 0}^{2}=z s / 4$ and the $\gamma \mathrm{p}$ cross section is computed assuming factorization of the hard-scatter cross section (evaluated in leading-order $Q \mathrm{QD}$ ) and the proton and photon structure functions.

Let us briefly discuss the main uncertainties implicit in the above prescription (for a more detailed discussion see [3]). The factorization of the impact parameter dependence is merely a plausible assumption, as is the expected shape of the function $A(b)$. The choice $P_{\mathrm{VMD}}=1 / 300$ and its factorization is a starting point for a more detailed analysis, certainly we insist that all factors of $\alpha_{t e n}$ be removed from the exponential in order to eikonalize correctly. The assumption that the individual hard scatters be incoherent is again a first step and may well be reasonable for such high parton densities. The QCD scale dependence is expected to be significant, especially at low $p_{\Upsilon}$ (where most of the activity is found): a full next-to-leading-order calculation ought to reduce this sensitivity.

Starting from this formalism, we move on next to discuss the method of event generation.

\section{Event generation}

Our strategy for event generation is now described. Assuming there to be at least one hard scatter, we use (4) with $n=1$ to generate the impact parameter for the collision and the $z$ of the photon. For $A(b)$ we use the expression of [4], and for $f_{y / \mathrm{c}}\left(z, E^{2}\right)$ we used the same (leading-logarithmic) Weizsäcker-Williams expression as in [3] with $E^{2}=z s / 4$. The differential probability is first assumed to factorize into three parts: a $b$-dependent part, a $z$-dependent part and a $p_{\mathrm{Tl}}$-dependent part. We can then choose $b, z$ and $p_{\mathrm{T} 1}$ and accept according to the correct distribution. It is then straightforward to pick $x_{\mathrm{pl}}$ and $x_{y \mathrm{I}}$.

Moving on to the second hard scatter, we first decide if there was a scatter in the range $p_{T \min } \leqslant p_{T 2} \leqslant p_{T 1}$, using the probability

$$
1-\exp \left(-\int_{p_{\text {TIP }}^{2}}^{p_{T 1}^{2}} \mathrm{~d} p_{\mathrm{T}}^{2} \frac{A(b)}{P_{\mathrm{VMD}}} \frac{\mathrm{d} \sigma^{\gamma \mathrm{p}}\left(p_{\mathrm{T}}^{2}, z s\right)}{\mathrm{d} p_{\mathrm{T}}^{2}}\right) .
$$

If there is no scatter, the event generation is terminated, otherwise we proceed to select the $P_{\text {T }}$ value according to the distribution

$$
\sim \frac{\mathrm{d} \sigma^{\gamma \mathrm{P}}}{\mathrm{d} p_{\mathrm{T}}^{2}} \exp \left(-\int_{p_{\mathrm{T}}^{2}}^{\rho_{\mathrm{T}}^{2}} \mathrm{~d} p_{\mathrm{T}}^{2} \frac{A(b)}{P_{\mathrm{VMD}}} \frac{\mathrm{d} \sigma^{\gamma \mathrm{P}}\left(p_{\mathrm{T}}^{2}, z s\right)}{\mathrm{d} p_{\mathrm{T}}^{2}}\right)
$$

where (as for the first scatter) we approximate this distribution by the differential cross section and accept according to the full distribution. Once $p_{\mathrm{T} 2}$ has been chosen, it is again 
straightforward to pick the parton longitudinal momenta. This procedure is then repeated for successive hard scatters until the chain is terminated as described above.

At the present time, we do not specify the final state parton types, and merely fragment assuming them all to be gluons. Also, we appreciate that the incoherent hard-scattering model used here is unable to address the issue of longitudinal momentum conservation, i.e. the requirement that $\sum x_{\mathrm{pi}}, \sum x_{y i} \leqslant 1$. It is necessary to include inter-parton correlations if one is to account correctly for this requirement. However, we expect this to be a smail effect given the predominance of relatively small- $x$ partons in multi-jet production. In practice, with $P_{\text {Tmin }}=2 \mathrm{GeV}$, failure to conserve momentum is around $3 \%$ of all events. We take a conservative approach (in the sense that it is expected to lead to a reduction in the number of multiple scattering events) and simply disregard those events which lead to a violation of momentum conservation.

To simulate the physics between the parton level and the hadronic final state, we have interfaced our generator to the HERWIG [6] generator. HERWIG simulates initial- and finalstate QCD radiation and uses a cluster hadronization model to produce final state hadrons. Gluon interference plays an important role in the QCD radiation, and it is thus necessary to specify the colour connection between initial- and final-state partons. We have proceoded as follows. In an event with a multiple scatter, the colour connections for the first hard scatter are treated as is determined by the cross section for the $s, t$ and $u$ channel $\mathrm{gg} \rightarrow \mathrm{gg}$ diagrams. The final-state partons from each subsequent hard scatter are then connected only to each other. Thus, for the first hard scatter initial-state QCD radiation is generated correctiy, whilst for subsequent hard scatters final-state QCD radiation is generated with simplified colour connections and initial-state radiation is not simulated at all. Unless stated explicitly in the following, the default parameters of HERWIG version 5.6 were used.

We used the GRV leading-order structure functions for the photon [7]. For the proton, we used the MRSD'. parametrization [8] converted to the DIS scheme (the parton distributions are then anticipated to be very close to these obtained from a leading order fit which should, strictiy speaking, be used) [9]. The QCD scale, $Q^{2}$, used to determine the structure functions and the scale of the strong coupling, is assumed to be the transverse momentum of the hard scatter, i.e. $p_{T}^{2}$.

\section{Results}

The study and simulation of hadronic final states is a complex topic. It is our intention here to point out briefly some of the gross features to be expected in events containing multiple parton scatters. Due to the uncertainty in the amount of double-counting between low- $p_{T}$ parton scattering and the 'soft underlying event' (as simulated in HERWIG) we restrict ourselves for now to those features observable at low absolute pseudorapidity $|\eta|$ (where $\eta=\ln (\tan (\theta / 2))$ and $\theta$ is the polar angle defined relative to the proton direction), without going into detailed examination of the region around the hadronic remnants. Here, we shall merely point out that, as is shown in figure 1, multiple scatters occur mainly at low transverse momentum and thus can be expected to have a large effect in the remnant regions. Certainly further study in the region close to the beam pipe is of interest, where multiple scattering can be expected to compete with the expected enhancement of initial-state radiation (at least partially due to semi-hard physics) to produce the observed enhancement of hadronic activity. In order to investigate the effect of multiple scattering, we generated 3000 events with a $p_{\mathrm{Tmin}}$ of $2 \mathrm{GeV}$ and with the $\gamma_{\mathrm{P}} \mathrm{CM}$ energy restricted to being above $0.5 \sqrt{s}$ (the HERA CM energy, $\sqrt{s}$, is equal to $296 \mathrm{GeV}$ for $26.7 \mathrm{GeV}$ electrons and $820 \mathrm{GeV}$ protons). The fraction of events containing more than one hard scatter is $20.4 \pm 0.9 \%$ (corresponding 
to a mean number of hard scatters per event of 1.30). For comparison, we generated a further sample of 3000 events with multiple scattering turned off.

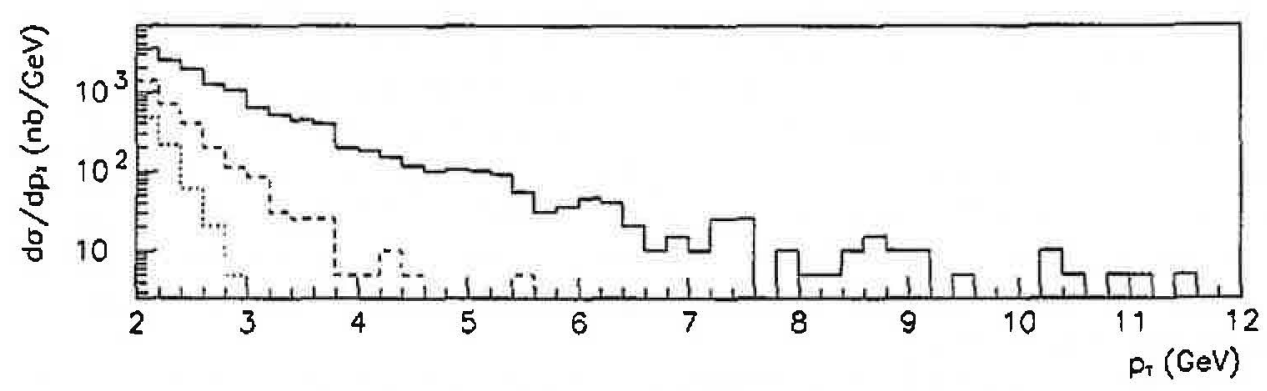

Figure 1. $p_{T}$ of hardest scatter (full histogram), second hardest (broken histogram) and third hardest scalter (dotted histogram) for photoproduction events at HERA.

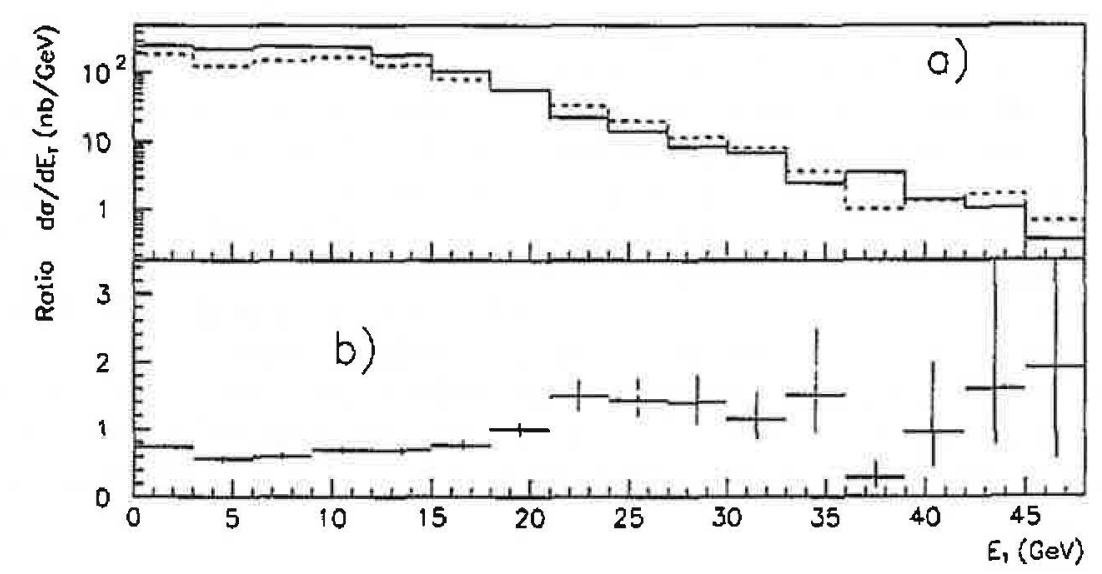

Figure 2. (a) Scalar transverse energy within the region $|\eta|<2$ for uneikonalized photoproduction events (full curve) and eikonalized events (broken curve)-normalized to ep cross section. (b) Shows the ratio of the histograms in (a).

Figure 2 shows the total scalar transverse energy $\left(E_{T}\right)$ within a pseudorapidity cut $|\eta|<2$, both with and without multiple scattering. Figure 3 shows likewise the number of charged particles in the same region of $|\eta|$. The ratios of the histograms for the two event samples are also shown, with statistical errors. We see a clear suppression at low values of $E_{\mathrm{T}}$ and multiplicity in the event sample when multiple scattering is tumed on, and a trend towards longer tails to high $E_{\mathrm{T}}$ and multiplicity.

For an event with a single hand scatter, we expect the energy within a rapidity cut $|\eta|<2$ to come principally from a single pair of back-to-back jets, defining an axis in the $X Y$ plane. However, if more than one hard scatter has taken place, more energy will be found away from this axis. To investigate this effect we have calculated the shape (or circularity) variable. To calculate the shape variable, we first find the principal $X Y$ axis of the event, $S$. This is the vector which maximizes $\sum\left|S \cdot p_{T}\right|$ where the sum is over all particles in the event satisfying the rapidity cut. The 'shape' is then the quantity $\sum\left|S \cdot p_{\mathrm{T}}\right|^{2} / \sum p_{\mathrm{T}}^{2}$, and is equal to one for an event consisting of two exactiy back-to-back particles, and one half for 


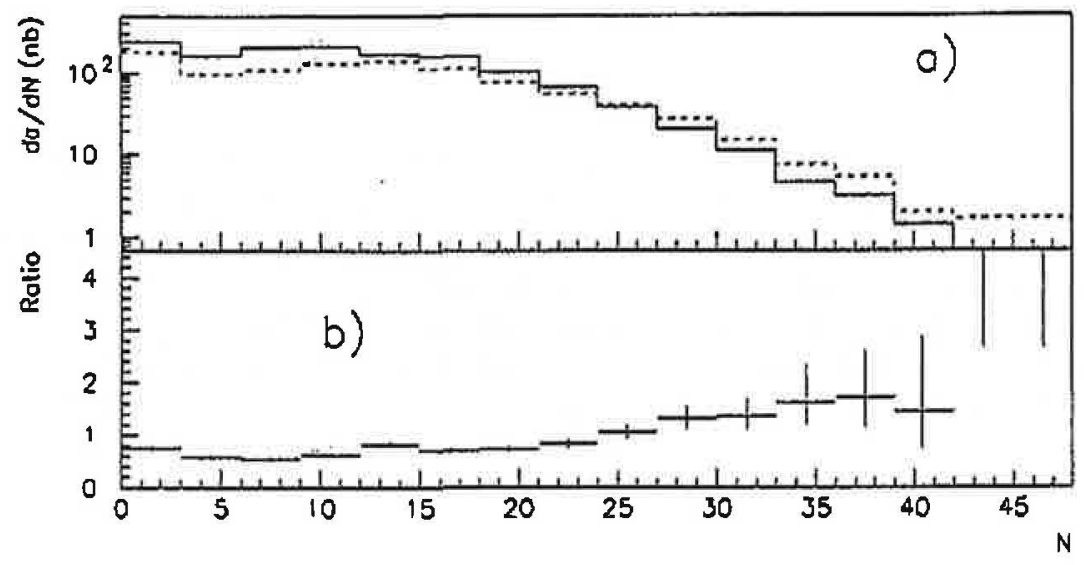

Figare 3. (a) Charged particle multiplicity within the region $|\pi|<2$ for uneikonalized photoproduction events (full curve) and eikonalized events (broken curve). Nomalized to ep cross section. (b) Shows the ratio of the histograms in (a).

a perfectly circular event, i.e. one with an isotropic distribution of energy in the azimuthal plane. Another quantity of interest is $E_{\mathrm{T}}(S)$ the scalar sum of energy flow along the axis $S$. In our model this will approximate twice the $p_{\mathrm{T}}$ of the hardest scatter in an event. In figure 4 we have plotted events with $E_{\mathrm{T}}(S)>6 \mathrm{GeV}$. We see that with multiple scattering tumed on, less events populate the high 'shape' region, while the high shape region shows signs of a slight excess.

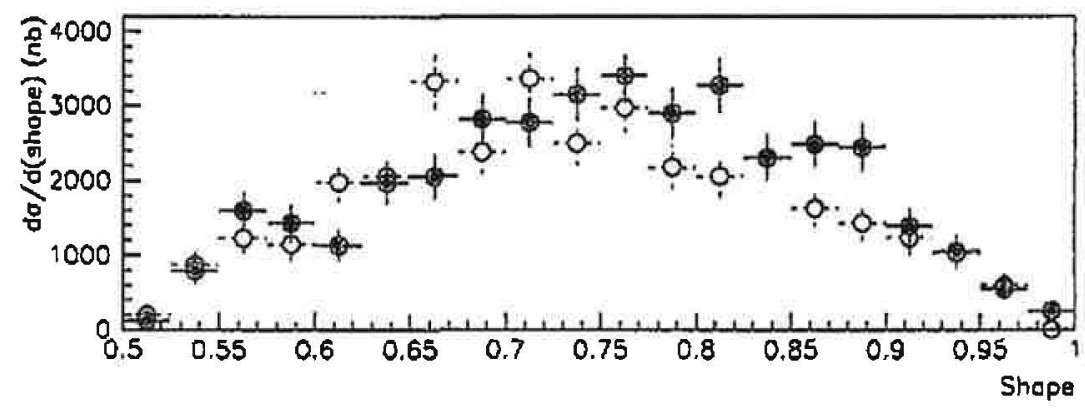

Figure 4. Shape variable for events with $E_{\mathrm{T}}(S)>6 \mathrm{GeV}$, uneikonalized (shaded circles) and eikonalized (open circles), normalized to ep cross sections.

It should be recognized that the effects shown here could perhaps be reproduced by some other combination of fragmentation effects and more study is required in order to isolate the effect of multiple scattering. A particularly interesting possibility is the identification of more than one pair of high- $p_{T}$ jets, each pair individually balanced in $p_{T}$. Specifically, we have computed the cross section for events with at least two hard scatters (i.e. four or more jets), both of which have $p_{\mathrm{T}} \geqslant 6 \mathrm{GeV}$, and find it to be equal to $0.37 \mathrm{nb}$. This offers the intriguing possibility of observing directly multiple hard scattering in a relatively unambiguous experimental and theoretical regime. 


\section{Conclusions}

The generator used here is still in a rather primitive state, and a number of improvements and extensions are to be made. In the short term these include the use of alternative eikonal models, proper inclusion of different parton types and the imposition of momentum conservation. Measures to increase the efficiency of event generation need also to be implemented, and these studies carried out with higher statistics. However, our first results suggest that as well as affecting QCD predictions for the total $\gamma p$ cross section, multiple scattering could have an effect on event shapes which would be observable in the detectors at HERA. Any event simulation using perturbative QCD to generate jets with $p_{T}$ as low as $\sim 3 \mathrm{GeV}$ at HERA energies should take the possibility of multiple parton interactions into account.

\section{Acknowledgments}

We should like to thank Dr J K Storrow and the other members of the Durham photoproduction working group for advice and encouragement. JRF would also like to thank the theoretical physics and ZEUS experimental groups at DESY for assistance during a visit. This work was supported in part by the US National Science Foundation and in part by the UK Science and Engineering Research Council.

\section{References}

[1] For a general review see Buctumüller W and Ingelman G (ed) 1992 Proc. Workshop Physics at HERA (DESY, Hamburg, 1991)

Gribov L V, Levin E M and Ryskin M G 1980 Phys. Rep. 100 I

[2] Collins J C and Ladinsky O A 1991 Phys. Rev. D 432847

Forshaw J R and Storrow J K 1991 Phys. Lett. 268B 116: 1992 Phys. Lett. 276B 565

[3] Forshaw J R and Storsow J K 1992 Phys. Kev. D 464955

[4] Fletcher R S. Gaisser T K and Halzen F 1992 Phys. Rev. D 45, 337; 1992 Phys. Rev. D 453279

[5] Sjöstrand T and van Zij] M 1987 Phys. Rev. D 362019

[6] Marchesini G et al 1992 C. Phys. Com. 67 456-508

[7] Gluck M, Reya E and Vogt A 1991 Dortmund University preprint DO-TH 91/26

[8] Martin A D, Roberts R G and Stirling W I 1993 Phys. Lett. 306145

[9] Roberts R G 1993 Private communication 